\title{
EAAI-10: The First Symposium on Educational Advances in Artificial Intelligence
}

\author{
Marie desJardins, Mehran Sahami, and Kiri Wagstaff
}

\begin{abstract}
- The first symposium on Educational Advances in Artificial Intelligence (EAAI) was held in July 2010 in conjunction with the AAAI Conference on Artificial Intelligence (AAAI-10). It included an invited talk, paper presentations, model AI assignments, a teaching and mentoring workshop, a best educational video award, and a robotics track. This report summarizes that symposium.
\end{abstract}

$\mathrm{T}$ The first symposium on Educational Advances in Artificial Intelligence (EAAI) was held on July 13-14, 2010, in conjunction with the AAAI Conference on Artificial Intelligence (AAAI-10). The symposium was established in response to growing community interest in sharing best practices for (1) how to teach AI and (2) how AI can serve as a motivating problem for teaching concepts in other areas of computer science, especially in introductory computer science courses. EAAI encourages the sharing of innovative educational approaches that convey or leverage AI and its many subfields, including robotics, machine learning, natural language, and computer vision. EAAI follows the successful 2008 Spring Symposium on "Using AI to Motivate Greater Participation in Computer Science" and the 2008 AAAI AI Education Colloquium. Fifty-five attendees formally registered for the event, but many other AAAI attendees also visited one or more EAAI events. EAAI is planned to become an annual event; EAAI- 11 will be held in San Francisco on August 9-10, 2011, collocated with AAAI-11.

The 2010 symposium included an invited talk, paper presentations, model AI assignments, a teaching and mentoring workshop, a best educational video award, and a robotics track.

The EAAI invited talk was presented by Mark Guzdial (Georgia Institute of Techology). His talk, titled "Technology for Teaching the Rest of Us," focused on how to teach computer science (or more broadly, computational thinking) to students who may not be computer science majors or may lack the background or motivation to do well in a traditional computer science course. The core message of the talk was about the need to 
identify a context in which computer science concepts (or programming) makes sense to the students, such as manipulating digital media in the form of images or video. These contexts have resulted in increased student dedication and retention and are a powerful way to engage and motivate students at every level. Contexts from the field of AI (such as robotics, learning, game playing, and natural language processing) may be particularly exciting and effective for students. Guzdial's talk set the tone for the rest of the symposium, with a combination of innovative pedagogical ideas and rampant enthusiasm for the subject.

Six full-length papers were presented at EAAI-10, covering a rich variety of AI teaching challenges and solutions. Topics included the use of Pacman as a motivating testbed for teaching AI, research related to the use of robots in education, and information retrieval as a motivating application for AI courses at the college level, as well as methods for making AI approachable and fun for elementary school students. Two posters (both on roboticsbased education) were also accepted for presentation in the main AAAI poster session.

The model AI assignments sessions of EAAI-10 presented eight innovative, ready-to-adopt course assignments that have been used successfully in a variety of AI courses. This program has its roots in the popular nifty assignments ${ }^{1}$ session at the ACM Special Interest Group on Computer Science Education (SIGCSE), but with a focus on AI-related assignments rather than projects aimed at introductory computer science courses. We have created a persistent online repository for the Model AI Assignments projects, ${ }^{2}$ from which educators may download pedagogical materials.

The second day of EAAI-10 was devoted to a half-day teaching and mentoring workshop. Our goal was to bring current and future AI educators together in an engaging, interactive setting to stimulate creativity in teaching strategies and to facilitate the creation of professional contacts and mentoring relationships. To achieve this goal, the workshop deliberately departed from a traditional presentation format.

Mehran Sahami (Stanford University) opened the workshop with an enthusiastic, entertaining presentation on "Creating Classroom Engagement through Active Learning." His talk engaged the attendees in an active learning exercise designed to build intuition about probability. Sahami emphasized the difference between teaching as a "sage on the stage" versus a "guide on the side" in increasing student understanding, motivation, engagement, and retention.

Next, attendees divided into small groups to brainstorm ideas for ways to engage students specifically when teaching difficult AI topics: decision trees, Bayesian networks, hidden Markov models, multiagent systems, reinforcement learning, and $A^{*}$ search. After 45 minutes of breakout discussion, each group presented their ideas to the workshop as a whole.

The workshop concluded with a panel focused on challenges in the classroom. The panelists were Marie desJardins (University of Maryland, Baltimore County), Zachary Dodds (Harvey Mudd College), and Michael Littman (Rutgers University). The discussion topics included start-time creep (including issues related to how to get students to arrive on time to class), the challenges in properly preparing (overpreparing versus underpreparing) for class, how to write gradeable exams, how to deal with plagiarism and cheating, how to combat the perception by some students that $\mathrm{AI}$ is a "throwaway" class, and how to make students accountable when assigning group or pair programming assignments.

The 2010 AI Video Competition, in conjunction with EAAI-10, included an award for the best educational video, recognizing the innovative and effective use of video as a teaching tool. This year, the award was given to Jonathan Mugan and Benjamin Kuipers for their video, The Qualitative Learner of Action and Perception (QLAP). ${ }^{3}$

EAAI and the education track of the AAAI Robotics Workshop and Exhibition reinforced each other in several ways. Most of the talks at the roboticsthemed session at EAAI featured speakers from the Robotics Workshop education track. By the same token, the workshop's education-track session invited all EAAI participants to attend, and several attendees took advantage of that opportunity. The interactions increased over the following two days of exhibitions. EAAI participants joined the Robotics Workshop demonstrations, competitions, and poster session during breaks in their schedule.

\section{Notes}

1. See nifty.stanford.edu.

2. See modelai.gettysburg.edu.

3. This video, and all of the videos in the competition, are available at aivideo.org.

\section{Acknowledgements}

We gratefully acknowledge financial support from the National Science Foundation, Stanford University, and Google, Inc.

Marie desJardins is an associate professor of computer science and engineering at the University of Maryland, Baltimore County.

Mehran Sahami is an associate professor (teaching) of computer science at Stanford University.

Kiri Wagstaff, who earned a PhD in computer science from Cornell University in 2002, is currently a member of the research staff at the Jet Propulsion Laboratory. She is also working on a master's degree in geology at the University of Southern California. 